\section{Impacto da qualidade da atenção primária à saúde na redução das internações por condições sensíveis}

\author{
The impact of primary healthcare and the \\ reduction of primary health care-sensitive \\ hospital admissions
}

Dayanna Mary de Castro 1 Veneza Berenice de Oliveira 1 Amanda Cristina de Souza Andrade 2 Mariângela Leal Cherchiglia 1 Alaneir de Fátima dos Santos 1

doi: 10.1590/0102-311X00209819

\section{Resumo}

O objetivo foi analisar a associação entre a qualidade da atenção primária à saúde (APS) nos municípios brasileiros e o número de internações por condições sensíveis à atenção primária. Trata-se de estudo ecológico, com análise de dados secundários de abrangência nacional. A qualidade da APS foi aferida com base na avaliação do Programa Nacional de Melhoria do Acesso e da Qualidade da Atenção Básica (PMAQ-AB). A análise foi realizada por meio de um modelo explicativo hierarquizado, tendo sido o número de internações por condições sensíveis à atenção primária no ano de 2014 considerado como variável dependente, e os dados sociodemográficos e os relacionados ao sistema de saúde como variáveis independentes. A medida de associação entre o número de hospitalizações e a qualidade da APS foi calculada por regressão binomial negativa com variância robusta e população total como offset, tendo sido considerada significância de 20\% na análise univariada e 5\% na multivariada. A quantidade média de internações por condições sensíveis à atenção primária no período analisado foi de 359,97 internações por município. A qualidade da APS mostrou associação negativa com o número de internações por condições sensíveis à atenção primária. Municípios com menor nível de qualidade (quartil 1) apresentaram 21,2\% mais de internações por condições sensiveis à atenção primária do que municípios mais bem avaliados (IC95\%: 1,09-1,34). Os resultados mostraram que a qualidade da APS do país teve impacto na redução das internações por condições sensíveis à atenção primária, mesmo em contextos de vulnerabilidade social.

Política de Saúde; Pesquisa sobre Serviços de Saúde; Atenção Primária à Saúde; Assistência Ambulatorial; Qualidade da Assistência à Saúde

\section{Correspondência}

D. M. Castro

Rua José Braga da Silva 291, apto 402, Nova Lima, MG 34002-053, Brasil.

dayannamary@yahoo.com.br

1 Universidade Federal de Minas Gerais, Belo Horizonte, Brasil.

2 Universidade Federal de Mato Grosso, Cuiabá, Brasil. 


\section{Introdução}

As internações por condições sensíveis à atenção primária são utilizadas em muitos países como um instrumento para avaliar os sistemas de saúde 1,2. No Brasil, após a criação da lista nacional de internações por condições sensíveis à atenção primária em 2008 3, esse indicador tem sido usado para avaliar o comportamento das internações hospitalares e sua associação com a Estratégia Saúde da Família (ESF), modelo brasileiro de atenção primária à saúde (APS) 4,5.

De fato, as evidências demonstram as repercussões positivas em indicadores de saúde em consequência da expansão da ESF, como a redução das mortalidades infantil, cardiovascular e cerebrovascular, da mortalidade em menores de cinco anos por diarreia e pneumonias, e das internações por condições sensíveis 6,7,8,9,10,11. Entretanto, somente o aumento da cobertura da APS pode não ser suficiente para reduzir as internações por condições sensíveis à atenção primária ${ }^{12}$. Nesse sentido, estudos mais amplos foram realizados e avaliaram, além da ampliação do número de equipes de APS, aspectos relacionados às características desses serviços, tais como a presença dos atributos da APS, a estrutura das unidades de saúde, processos de trabalho, características e formação dos profissionais $4,13,14,15,16,17,18,19$.

No Reino Unido, crianças com vacinação incompleta e que não realizavam consultas para acompanhamento do desenvolvimento apresentaram risco maior de internação sensível ${ }^{15}$. Ademais, um estudo com beneficiários idosos do Medicare (Estados Unidos), um programa de plano de saúde americano que assiste essencialmente indivíduos com 65 anos ou mais, mostrou que pessoas menos satisfeitas com a coordenação e a qualidade do cuidado recebido eram mais propensas a internações por condições sensíveis à atenção primária do que aquelas completamente satisfeitas 16 . Outros estudos também evidenciaram que a continuidade do cuidado, a acessibilidade dos serviços e a melhoria da qualidade focada na APS são fatores associados à redução de hospitalizações potencialmente evitáveis $2,17,20$.

No Brasil, estudos recentes que avaliaram aspectos da estrutura e processo de trabalho na APS encontraram associação entre essas características e a ocorrência de internações por condições sensíveis à atenção primária. Araújo et al. 13 concluíram que o horário de funcionamento das unidades de saúde e a disponibilidade de vacinas são fatores que reduzem o número de internações por condições sensíveis à atenção primária. No Estado de Santa Catarina, para municípios de médio e grande porte, as variáveis "presença de médico pediatra na unidade de saúde" e "acesso ao sistema de saúde" apresentaram associação inversa ao risco de hospitalização por condições sensíveis à atenção primária em menores de cinco anos de idade. Em municípios de pequeno porte, "equipe de Saúde da Família completa" e "consulta de puericultura" também foram variáveis com associação inversa ao risco de internações por condições sensíveis à atenção primária 14 .

Em relação à avaliação da qualidade da APS no Brasil, destaca-se o Programa Nacional de Melhoria do Acesso e Qualidade da Atenção Básica (PMAQ-AB), um programa de abrangência nacional lançado em 2011 pelo Ministério da Saúde, que certifica o desempenho das equipes de APS com base em inúmeros padrões de qualidade, sendo, portanto, um marcador do nível de acesso e qualidade da assistência prestada ao usuário na APS 21. No ano de 2014, o PMAQ-AB avaliou 30.522 equipes em mais de $90 \%$ dos municípios brasileiros.

Nesse sentido, frente à indagação se municípios com maior nível de qualidade da APS têm menos internações por condições sensíveis, o objetivo deste estudo foi investigar a associação entre a qualidade da atenção primária nos municípios brasileiros e o número de internações por condições sensíveis.

\section{Materiais e métodos}

Trata-se de um estudo ecológico, com análise de dados secundários de abrangência nacional. A unidade de análise referiu-se aos municípios brasileiros que participaram do segundo ciclo do PMAQ-AB no ano de 2014. Devido à adesão das equipes de APS ao PMAQ-AB ser voluntária, realizou-se o cálculo do percentual das equipes que aderiram ao Programa por município. Foram excluídos da análise os municípios com menos de $80 \%$ de adesão de equipes de saúde. A amostra final do estudo contou 
com a inclusão de 69,9\% dos municípios do país $(\mathrm{n}=3.897)$. Características relevantes dos municípios incluídos e excluídos foram comparadas por meio do teste de Mann-Whitney e teste qui-quadrado, sem diferença significativa entre os grupos.

O desfecho foi o número de internações por condições sensíveis à atenção primária por local de residência, com a data de alta no ano de 2014, obtido por meio do Sistema de Informações Hospitalares do Sistema Único de Saúde (SIH-SUS). Entre 6.304.146 hospitalizações (excluindo-se partos), 1.402.810 internações por condições sensíveis à atenção primária foram detectadas utilizando-se a lista de 20 grupos de doenças derivadas da décima revisão da Classificação Internacional de Doenças (CID-10) e validadas como condições sensíveis à atenção primária no Brasil.

A variável explicativa principal referiu-se à qualidade da APS municipal. Essa variável foi construída baseando-se nas notas provenientes da avaliação das equipes de APS que participaram do segundo ciclo do PMAQ-AB, extraídas da base de dados nacional do Programa. Essa base contém as notas alcançadas por cada equipe no somatório das etapas do Programa: autoavaliação, análise dos indicadores e avaliação externa. Essas notas podem variar de 0 a 100.

A avaliação do PMAQ-AB teve como unidade de análise a equipe. Contudo, como a unidade de análise do presente estudo referiu-se a municípios brasileiros, foi necessário construir um indicador agregado para o nível municipal com base na nota das equipes avaliadas pelo PMAQ-AB. A variável "qualidade da atenção básica municipal" foi, portanto, obtida por meio da média das notas das equipes submetidas à avaliação do PMAQ-AB. Para as análises uni e multivariada, a "qualidade da atenção básica municipal” foi estratificada em quartis pela média de cada município, considerando-se o quartil 1 como o menor nível de qualidade e o quartil 4 como o maior nível de qualidade da APS.

As demais variáveis independentes foram selecionadas baseando-se em evidências que apontaram sua relação com o desfecho em estudo 5,13,22,23,24. Todas as variáveis usadas neste estudo estão apresentadas no Quadro 1.

\section{Quadro 1}

Descrição das variáveis independentes e dependentes.

\begin{tabular}{|c|c|c|}
\hline Variáveis & Descrição & Fonte \\
\hline$\%$ de menores de 5 anos na população & $\begin{array}{l}\text { Percentual de pessoas com menos de } 5 \text { anos } \\
\text { de idade em relação à população total, em } \\
\text { determinado município, no ano considerado. }\end{array}$ & $\begin{array}{l}\text { Estimativa populacional por município, sexo e } \\
\text { idade-RIPSA *, ano } 2014 .\end{array}$ \\
\hline \% de idosos na população & $\begin{array}{l}\text { Percentual de pessoas com } 60 \text { anos de idade } \\
\text { ou mais em relação à população total, em } \\
\text { determinado município, no ano considerado. }\end{array}$ & $\begin{array}{l}\text { Estimativa populacional por município, sexo e } \\
\text { idade - RIPSA *, ano } 2014 .\end{array}$ \\
\hline Índice de vulnerabilidade social & $\begin{array}{l}\text { Medida composta de } 16 \text { indicadores organizados } \\
\text { em três dimensões da vulnerabilidade social: } \\
\text { infraestrutura urbana; capital humano dos } \\
\text { domicílios; renda, acesso ao trabalho e forma } \\
\text { de inserção (formal ou não) dos residentes. Os } \\
\text { municípios foram classificados segundo níveis de } \\
\text { vulnerabilidade: baixo, médio, alto. }\end{array}$ & Atlas da Vulnerabilidade Social/IPEA **, ano 2010. \\
\hline Porte municipal & $\begin{array}{c}\text { Classificação dos municípios segundo sua } \\
\text { população: pequeno porte I - até } 20.000 \\
\text { habitantes; pequeno porte II - de } 20.001 \text { a } \\
50.000 \text { habitantes; médio porte - de } 50.001 \text { a } \\
100.000 \text { habitantes; grande porte - de } 100.001 \text { a } \\
900.000 \text { habitantes; metrópole - mais de } 900.000 \\
\text { habitantes. }\end{array}$ & $\begin{array}{l}\text { Estimativa populacional por município, sexo e } \\
\text { idade-RIPSA *, ano } 2014 .\end{array}$ \\
\hline
\end{tabular}

(continua) 
Quadro 1 (continuação)

\begin{tabular}{|c|c|c|}
\hline Variáveis & Descrição & Fonte \\
\hline$\%$ de cobertura de ESF & $\begin{array}{l}\text { Cobertura populacional de equipes de saúde da } \\
\text { família no município, obtida pelo cálculo: número } \\
\text { de equipes de Saúde da Família implantadas } \\
\text { × 3.450/população, multiplicado por } 100 \text {. } \\
\text { Limitada a 100\%. Com base no cálculo descrito, o } \\
\text { município recebe a seguinte classificação (Costa } \\
\text { \& Calvo 35): Baixa cobertura: até } 49,9 \% \text {; Média } \\
\text { cobertura: entre } 50 \text { a } 69,9 \% \text {; Alta cobertura: } \\
\text { maior ou igual a } 70 \% \text {. }\end{array}$ & $\begin{array}{l}\text { Departamento de Atenção Básica } \\
\text { do Ministério da Saúde, ano } 2014 .\end{array}$ \\
\hline $\begin{array}{l}\% \text { de cobertura de planos privados de } \\
\text { saúde }\end{array}$ & $\begin{array}{c}\text { Razão entre número de vínculos de beneficiários } \\
\text { de planos e seguros privados de saúde e a } \\
\text { população, multiplicado por } 100 \text {. }\end{array}$ & $\begin{array}{l}\text { Agência Nacional de Saúde Suplemantar, } \\
\text { ano } 2014 .\end{array}$ \\
\hline Número de leitos por habitante & $\begin{array}{l}\text { Número de leitos hospitalares conveniados } \\
\text { ou contratados pelo SUS, por mil habitantes } \\
\text { residentes, em determinado município, no ano } \\
\text { considerado. }\end{array}$ & $\begin{array}{l}\text { Cadastro Nacional de Estabelecimentos de } \\
\text { Saúde, ano } 2014 .\end{array}$ \\
\hline $\begin{array}{l}\text { Número de internações por condições } \\
\text { sensíveis à atenção primária }\end{array}$ & $\begin{array}{l}\text { Número absoluto de internações por condições } \\
\text { sensíveis à atenção primária. }\end{array}$ & SIH-SUS, ano 2014. \\
\hline $\begin{array}{l}\text { Coeficiente de internações por } \\
\text { condições sensíveis à atenção primária }\end{array}$ & $\begin{array}{l}\text { Razão entre o número de internações por } \\
\text { condições sensíveis à atenção primária e } \\
\text { a população do município a cada } 10.000 \\
\text { habitantes. }\end{array}$ & SIH-SUS, ano 2014. \\
\hline $\begin{array}{l}\text { Proporção de internações por condições } \\
\text { sensíveis à atenção primária }\end{array}$ & $\begin{array}{c}\text { Percentual de internações por condições } \\
\text { sensíveis à atenção primária em relação ao total } \\
\text { de internações (exceto parto), em determinado } \\
\text { município, no período considerado. }\end{array}$ & SIH-SUS, ano 2014. \\
\hline Qualidade da atenção básica municipal & $\begin{array}{l}\text { Média da nota da avaliação das equipes de } \\
\text { saúde da família e equipes de atenção básica } \\
\text { participantes do segundo ciclo do PMAQ-AB, em } \\
\text { determinado município. }\end{array}$ & Banco de dados do 2 o ciclo do PMAQ-AB. \\
\hline
\end{tabular}

ESF: Estratégia Saúde da Família; IPEA: Instituto de Pesquisa Econômica Aplicada; PMAQ-AB: Programa Nacional de Melhoria do Acesso e Qualidade da Atenção Básica; RIPSA: Rede Interagencial de Informações para à Saúde; SIH-SUS: Sistema de Informações Hospitalares do Sistema Único de Saúde.

Fonte: elaboração dos autores.

* http://tabnet.datasus.gov.br/cgi/deftohtm.exe?novapop/cnv/popbr.def (acessado em 01/Mar/2018);

** http://ivs.ipea.gov.br/index.php/pt/planilha (acessado em 01/Mar/2018).

Ainda, foi realizada análise descritiva das variáveis independentes, apresentando a frequência e a proporção para as variáveis categóricas. Para as variáveis contínuas, foram utilizadas média, mediana, desvio padrão, mínimo, máximo e quartis. Também foi feita a análise descritiva do coeficiente e proporção de internações por condições sensíveis à atenção primária. As análises simples e múltipla foram realizadas usando-se o modelo de regressão binomial negativa a fim de solucionar a superdispersão dos dados. O logaritmo da população foi incluído como offset, o que permitiu estimar as medidas de associação sobre a taxa.

Para a modelagem, utilizou-se a análise hierarquizada em dois blocos. O bloco distal correspondeu às variáveis sociodemográficas, e o bloco proximal apresentou variáveis relacionadas aos serviços de saúde dos municípios. $\mathrm{Na}$ análise ajustada, inicialmente, todas as variáveis do bloco distal foram incluídas no modelo. A inclusão de novas variáveis em cada bloco foi realizada pelo método backward, tendo permanecido no modelo as variáveis associadas com nível de significância menor que 0,20 . As 
estimativas da regressão e o intervalo de 95\% de confiança (IC95\%) foram verificados no bloco correspondente, e o valor de $\mathrm{p} \leq 0,05$ foi considerado para as associações significativas. Para a análise estatística e processamento dos dados, foi utilizado o software Stata versão 12.0 (https://www.stata.com).

\section{Resultados}

O número absoluto, o coeficiente e a proporção de internações por condições sensíveis à atenção primária, geral e por estratos de idade, estão descritos na Tabela 1. No ano de 2014, o número médio de internações por condições sensíveis à atenção primária foi de 359,97 por município, com variação entre 2 e 85.139. A média do coeficiente de internações por condições sensíveis à atenção primária foi de 151,76 (desvio padrão - DP = 120,27). Analisando por faixas etárias, o coeficiente de internações por condições sensíveis à atenção primária de idosos foi quase seis vezes maior que o da população entre 5 e 59 anos (média de 510,62 e 87,49, respectivamente). As internações sensíveis representaram, em média, $24,7 \%$ do total de internações dos municípios no ano de 2014, excluídos os partos.

As Tabelas 2 e 3 apresentam as características das variáveis independentes. A proporção de crianças menores de cinco anos nos municípios do estudo teve média de 7,25\% (DP $=1,78)$. Já o porcentual médio de idosos foi de $12,94 \%$ ( $\mathrm{DP}=3,71)$. A cobertura média de ESF foi de $88,52 \%(\mathrm{DP}=21,02)$ e $3.250(83,4 \%)$ municípios apresentaram uma alta cobertura. A cobertura média de planos privados de saúde nos municípios foi de $8,43 \%(\mathrm{DP}=10,59)$. O número de leitos por cada mil habitantes variou de 0 a 28,14, com média de 1,66 ( $\mathrm{DP}=2,10)$. Quanto ao porte, 3.498 (89,8\%) municípios apresentaram população inferior a 50 mil habitantes, tendo sido classificados como pequeno porte I $(\mathrm{n}=2.779$; $\mathrm{N}(\%)=71,3)$ e pequeno porte II $(\mathrm{n}=719 ; \mathrm{N}(\%)=18,5)$. O índice de vulnerabilidade social (IVS) foi considerado alto em $1.272(32,6 \%)$ municípios. Quanto à qualidade da ESF medida pela nota da avaliação do PMAQ-AB, apenas 975 (25\%) dos municípios avaliados tiveram nota maior que 60,5. A nota máxima alcançada, em uma escala de 0 a 100, foi de 80,54, com média de 53,43 ( $\mathrm{DP}=10,11$ ).

Os resultados dos modelos inicial e final estão demonstrados na Tabela 4. Na análise univariada, apresentaram associação inversa significativa o porte municipal, o percentual de planos de saúde e a qualidade da atenção básica. Na análise ajustada, no bloco distal, municípios com maior proporção de idosos e maior vulnerabilidade social apresentaram maiores coeficientes de internações por condi-

Tabela 1

Análise descritiva das internações por condições sensíveis à atenção primária nos municípios participantes do 2o ciclo do Programa Nacional de Melhoria do Acesso e Qualidade da Atenção Básica (PMAQ-AB) *. Brasil, 2014.

\begin{tabular}{|c|c|c|c|c|c|c|c|}
\hline & Média & DP & Mínimo & Quartil 1 & Mediana & Quartil 3 & Máximo \\
\hline \multicolumn{8}{|l|}{ Número de internações por condições sensíveis à atenção primária } \\
\hline População geral & 359,97 & $1.585,82$ & 2,0 & 49,0 & 130,0 & 364,0 & 85.139 \\
\hline População < 5 anos & 44,96 & 238,22 & 0,0 & 4,0 & 11,0 & 34,0 & 11.547 \\
\hline População de 5-59 anos & 167,53 & 738,05 & 1,0 & 19,0 & 55,0 & 166,5 & 39.709 \\
\hline População $\geq 60$ anos & 147,48 & 623,97 & 0,0 & 23,0 & 57,0 & 154,0 & 33.883 \\
\hline \multicolumn{8}{|l|}{$\begin{array}{l}\text { Coeficiente de internações por condições sensíveis à atenção } \\
\text { primária }\end{array}$} \\
\hline População geral & 151,76 & 120,27 & 1,38 & 70,55 & 117,13 & 196,80 & $1.251,97$ \\
\hline População < 5 anos & 211,40 & 234,61 & 0,0 & 71,94 & 136,78 & 266,18 & $4.432,98$ \\
\hline População de 5-59 anos & 87,49 & 84,59 & 1,20 & 36,60 & 62,13 & 108,39 & 945,23 \\
\hline População $\geq 60$ anos & 510,62 & 341,72 & 0,0 & 266,11 & 433,67 & 669,76 & $3.011,69$ \\
\hline Proporção de internações por condições sensíveis à atenção primária & 24,70 & 11,18 & 3,61 & 16,56 & 22,36 & 30,39 & 81,59 \\
\hline
\end{tabular}

DP: desvio padrão.

Fonte: elaboração dos autores.

* Referente aos 3.897 municípios com adesão ao PMAQ-AB > 80\%. 
Tabela 2

Características descritivas das variáveis contínuas. Municípios participantes do 2o ciclo do Programa Nacional de Melhoria do Acesso e Qualidade da Atenção Básica (PMAQ-AB) *. Brasil, 2014.

\begin{tabular}{|c|c|c|c|c|c|c|c|}
\hline Variáveis & Média & DP & Mínimo & Quartil 1 & Mediana & Quartil 3 & Máximo \\
\hline$\%$ população $<5$ anos & 7,25 & 1,78 & 0,72 & 6,09 & 7,07 & 8,27 & 18,62 \\
\hline$\%$ população $\geq 60$ anos & 12,94 & 3,71 & 2,35 & 10,43 & 12,75 & 15,15 & 29,15 \\
\hline Qualidade da atenção primária à saúde & 53,43 & 10,11 & 17,55 & 46,60 & 53,75 & 60,50 & 80,54 \\
\hline Cobertura da Estratégia Saúde da Família & 88,52 & 21,02 & 0,71 & 85,81 & 100,0 & 100,0 & 100,0 \\
\hline Leitos/1.000 habitantes & 1,66 & 2,10 & 0,0 & 0,0 & 1,24 & 2,51 & 28,14 \\
\hline Cobertura de planos & 8,43 & 10,59 & 0,0 & 1,34 & 4,02 & 11,52 & 91,32 \\
\hline
\end{tabular}

DP: desvio padrão.

Fonte: elaboração dos autores.

* Referente aos 3.897 municípios com adesão ao PMAQ-AB > 80\%.

Tabela 3

Características descritivas das variáveis categóricas. Municípios participantes do 2o ciclo do Programa Nacional de Melhoria do Acesso e Qualidade da Atenção Básica (PMAQ-AB) *. Brasil, 2014.

\begin{tabular}{|c|c|c|}
\hline Variáveis & $\mathbf{n}$ & $\%$ \\
\hline \multicolumn{3}{|l|}{ Porte municipal } \\
\hline Pequeno porte I & 2.779 & 71,3 \\
\hline Pequeno porte II & 719 & 18,5 \\
\hline Médio porte & 219 & 5,6 \\
\hline Grande porte & 173 & 4,4 \\
\hline Metrópole & 07 & 0,2 \\
\hline \multicolumn{3}{|c|}{ Índice de vulnerabilidade social } \\
\hline Baixo & 1.706 & 43,8 \\
\hline Médio & 918 & 23,6 \\
\hline Alto & 1.272 & 32,6 \\
\hline \multicolumn{3}{|c|}{ Cobertura da Estratégia Saúde da Família } \\
\hline Baixa & 308 & 7,9 \\
\hline Média & 339 & 8,7 \\
\hline Alta & 3.250 & 83,4 \\
\hline
\end{tabular}

Fonte: elaboração dos autores.

* Referente aos 3.897 municípios com adesão ao PMAQ-AB > 80\%.

ções sensíveis à atenção primária. O número de internações sofreu um aumento de $8 \%$ a cada aumento de 1\% da população acima de 60 anos (IC95\%: 1,07-1,10). O número de internações por condições sensíveis à atenção primária em municípios com IVS alto foi 1,53 vez o número de internações por condições sensíveis à atenção primária em municípios com IVS baixo (IC95\%: 1,32-1,78). No bloco proximal, com exceção da cobertura de planos que não foi mantida no modelo final, todas as variáveis analisadas mostraram associação significativa com o desfecho do estudo. A cada aumento de uma unidade no número de leitos por mil habitantes, o número de internações por condições sensíveis à atenção primária aumentou 3,8\% (IC95\%: 1,02-1,06). Observou-se que municípios com maior cobertura de ESF registraram maior número de internações por condições sensíveis à atenção primária. Municípios com cobertura alta apresentaram 1,71 vez o número de internações de municípios com cobertura baixa (IC95\%: 1,51-1,93). A qualidade da APS mostrou associação negativa com o número 
Tabela 4

Análises simples e múltipla dos fatores associados às internações por condições sensíveis à atenção primária. Brasil, 2014.

\begin{tabular}{|c|c|c|c|c|c|c|}
\hline \multirow[t]{2}{*}{ Variáveis } & \multicolumn{3}{|c|}{ Análise simples } & \multicolumn{3}{|c|}{ Análise múltipla } \\
\hline & IRR & Valor de $p$ & IC95\% & IRR & Valor de p & IC95\% \\
\hline \multicolumn{7}{|l|}{ Bloco I } \\
\hline$\%$ população $<5$ anos & 0,997 & 0,877 & $0,956-1,039$ & & & \\
\hline$\%$ população $\geq 60$ anos & 1,058 & $<0,001$ & $1,042-1,075$ & 1,081 & $<0,001$ & $1,067-1,095$ \\
\hline \multicolumn{7}{|l|}{ Porte municipal } \\
\hline Pequeno porte I & 1,000 & & & & & \\
\hline Pequeno porte II & 0,801 & $<0,001$ & $0,760-0,843$ & & & \\
\hline Médio porte & 0,593 & $<0,001$ & $0,549-0,640$ & & & \\
\hline Grande porte & 0,427 & $<0,001$ & $0,398-0,458$ & & & \\
\hline Metrópole & 0,426 & $<0,001$ & $0,371-0,488$ & & & \\
\hline \multicolumn{7}{|c|}{ Índice de vulnerabilidade social } \\
\hline Baixo & 1,000 & & & & & \\
\hline Médio & 1,033 & 0,681 & $0,883-1,209$ & 1,238 & 0,011 & $1,051-1,459$ \\
\hline Alto & 1,263 & 0,001 & $1,103-1,446$ & 1,532 & $<0,001$ & $1,322-1,776$ \\
\hline \multicolumn{7}{|l|}{ Bloco II } \\
\hline Leitos/1.000 habitantes & 1,03 & 0,007 & $1,008-1,052$ & 1,038 & $<0,001$ & $1,020-1,056$ \\
\hline Cobertura de planos & 0,984 & $<0,001$ & $0,982-0,986$ & & & \\
\hline \multicolumn{7}{|c|}{ Cobertura da Estratégia Saúde da Família } \\
\hline Baixa & 1,000 & & & & & \\
\hline Média & 1,247 & 0,001 & $1,101-1,411$ & 1,217 & 0,003 & 1,068-1,387 \\
\hline Alta & 1,799 & $<0,001$ & $1,626-1,990$ & 1,709 & $<0,001$ & $1,514-1,929$ \\
\hline \multicolumn{7}{|c|}{ Qualidade da atenção primária à saúde } \\
\hline Quartil 4 & 1,000 & & & & & \\
\hline Quartil 3 & 1,147 & 0,157 & $0,949-1,386$ & 1,064 & 0,299 & $0,947-1,195$ \\
\hline Quartil 2 & 1,121 & 0,253 & $0,921-1,365$ & 1,136 & 0,015 & $1,025-1,259$ \\
\hline Quartil 1 & 1,202 & 0,065 & $0,989-1,462$ & 1,21 & $<0,001$ & $1,095-1,341$ \\
\hline
\end{tabular}

IC95\%: intervalo de 95\% de confiança; IRR: razão de taxas de incidência.

Fonte: elaboração dos autores.

de internações por condições sensíveis à atenção primária. Municípios com menor nível de qualidade (quartil 1) registraram 21,2\% mais internações por condições sensíveis à atenção primária do que aqueles mais bem avaliados (IC95\%: 1,09-1,34).

\section{Discussão}

No presente estudo, após ajuste para variáveis sociodemográficas e de características do sistema de saúde, a qualidade da atenção básica dos municípios brasileiros mostrou associação significativa com as internações por condições sensíveis à atenção primária. $\mathrm{O}$ número de internações por condições sensíveis à atenção primária nos municípios com o menor nível de qualidade foi $21,2 \%$ maior que nos municípios com o melhor nível.

A relação entre hospitalizações e a qualidade da APS tem sido estudada por vários autores e em distintos sistemas de saúde, e tem indicado que altas taxas de internações por condições sensíveis à atenção primária revelam uma baixa qualidade na APS 1,11,15,16,17.

A APS conta com recursos tecnológicos comprovadamente eficazes para realizar prevenção, além de fazer diagnósticos e intervenções precoces em vários agravos, reduzindo as incidências destas 
patologias e/ou suas complicações. Doenças infecciosas ou casos agudos podem ser prevenidos ou ter suas complicações controladas pela APS. Intervenções simples de educação em saúde realizadas pela ESF são capazes de reduzir as taxas de hospitalizações por doenças, tais como infecções de rim e trato urinário e desidratação. Muitas dessas patologias, mesmo se não forem prevenidas, podem ser manejadas ambulatorialmente e apenas por falta de assistência oportuna podem levar à complicação, requerendo internação. Pessoas com doenças crônicas ou multimorbidade, por sua vez, devem ter um acompanhamento regular pela APS, evitando complicações agudas que levem à internação 5,11,22.

Ações relacionadas à vacinação, consultas programadas para acompanhamento de certos grupos e atributos da APS, como coordenação do cuidado, longitudinalidade e acessibilidade têm sido associados à redução do risco de se internar por condições sensíveis à atenção primária 2,13,14,15,16,17,20. Contudo, muitos desses estudos avaliaram aspectos isolados da organização, estrutura e processo de trabalho na APS, sem o estabelecimento de uma classificação geral de qualidade dos serviços primários de atenção.

No Brasil, dois estudos aferiram a qualidade da APS utilizando um instrumento denominado Primary Care Assessment Tools (PCATools). O questionário PCATools foi desenvolvido para a avaliação da qualidade e da adequação da APS por meio dos seus atributos, tendo sido previamente validado em diferentes países, incluindo o Brasil 4 . Ambos os estudos não encontraram impacto de um maior nível de qualidade no risco de hospitalização por condição sensível. Contudo, em um deles, a classificação de qualidade das equipes avaliadas foi muito homogênea, dificultando a detecção de diferenças sutis de qualidade entre diferentes centros de saúde 4 . No outro, a qualidade das equipes avaliadas representou o nível mínimo necessário para fornecer uma atenção qualificada, mostrando que a qualidade dos serviços de saúde, se em nível baixo, não é capaz de influenciar as taxas de internações por condições sensíveis à atenção primária 18 .

De fato, isso reforça a importância de se usar a avaliação do PMAQ-AB como um instrumento de medida da qualidade da APS, especialmente na busca de uma relação com indicadores de morbidade hospitalar, como as internações por condições sensíveis à atenção primária. O PMAQ-AB proporciona ampla avaliação da APS em todo o território nacional, refletindo, portanto, o nível de qualidade da assistência prestada ao usuário no nível primário de atenção.

O coeficiente e o número de internações por condições sensíveis à atenção primária apresentaram grande dispersão nos municípios. Uma revisão sistemática da literatura internacional investigou as causas da variação geográfica nas internações por condições sensíveis à atenção primária, que foi evidenciada em $91 \%$ dos estudos analisados, sendo que em $64 \%$ destes a variação foi significativa. Fatores como facilidade no acesso à atenção secundária e inadequação da qualidade assistencial ou acesso na APS foram os principais fatores atribuídos às variações observadas 25. No Brasil, as já reconhecidas diversidades cultural, social e econômica também podem ser fatores explicativos para a grande dispersão dos coeficientes e números nos municípios estudados.

$\mathrm{Na}$ mesma direção do que já foi evidenciado na literatura, o estudo mostrou que características sociodemográficas exercem influência na ocorrência de internações por condições sensíveis à atenção primária. Crianças e idosos, por estarem nos extremos da vida, são mais susceptíveis ao agravamento de doenças, tanto agudas quanto crônicas, justificando a maior ocorrência de internações por condições sensíveis à atenção primária nestas faixas etárias 9,26. O IVS também manteve associação significativa com a ocorrência de internações por condições sensíveis à atenção primária, corroborando estudos nacionais e internacionais que identificaram maiores taxas de internações sensíveis na população de pior condição socioeconômica 9,20,23,24,27. Contudo, estudos já mostraram que sistemas de saúde universais e organizados por meio da APS podem suavizar a relação entre internações por condições sensíveis à atenção primária e vulnerabilidade social 28,29.

No que se refere às características do sistema de saúde, a média de leito por mil habitantes foi um fator de risco para internações por condições sensíveis à atenção primária. Associações positivas entre a disponibilidade de leitos de internação e taxas de internações por condições sensíveis à atenção primária foram detalhadas em outros estudos 20,25,30.

Em geral, a literatura mostra que a ampliação do acesso a serviços de APS parece estar associada à redução das internações por condições sensíveis à atenção primária 1,26,31,32, com algumas discordâncias 12,13,27,33. Neste estudo, a cobertura de ESF mostrou associação com o maior número de internações por condições sensíveis à atenção primária nas análises simples e múltipla, sugerindo que 
aspectos como o IVS e a qualidade da APS exercem mais influência nas internações sensíveis que a cobertura dos serviços.

O estudo apresentou algumas limitações, como o fato de ter sido construída uma medida de "nível de qualidade” da APS nos municípios brasileiros com base na avaliação das equipes de APS. Esse desenho pode não captar completamente as situações de heterogeneidade das equipes dentro de um município. Uma vez que a unidade de análise utilizada na avaliação do PMAQ-AB foi a equipe, outros modelos mais robustos podem ser usados para considerar e ponderar as diferenças dessas equipes em um mesmo município. Outra limitação do estudo foi a análise pontual do número de internações por condições sensíveis à atenção primária. Pesquisas que demonstraram associação da oferta de serviços de APS e internações sensíveis o fizeram por meio de análise de série temporal.

A inovação deste estudo, ao considerar a nota final da avaliação do PMAQ-AB como medida de qualidade da APS, ampliou o escopo da abordagem das internações por condições sensíveis à atenção primária, pois analisou a associação das internações com características do sistema de saúde que vão além da simples cobertura de ESF ou aspectos pontuais da estrutura e processo de trabalho das unidades básicas de saúde (UBS).

Entretanto, em 2020, após nove anos de sua criação e três ciclos, o PMAQ-AB foi finalizado com a publicação da Portaria no 2.979 34, de 12 de novembro de 2019. A Portaria institui o Programa Previne Brasil, que estabelece novo modelo de financiamento de custeio da APS. Uma das modalidades de financiamento do Programa é o "pagamento por desempenho". Segundo a Portaria, o incentivo financeiro será efetuado considerando-se os resultados de indicadores alcançados pelas equipes. Tais indicadores serão categorizados em indicadores de processo e resultados intermediários das equipes, indicadores de resultados em saúde e indicadores globais de APS. O Ministério da Saúde informou que a seleção dos indicadores considera a relevância clínica e epidemiológica, disponibilidade, simplicidade, baixo custo de obtenção, adaptabilidade, estabilidade, rastreabilidade e representatividade. O monitoramento será quadrimestral, com a granularidade por equipe 34. Assim, o futuro da avaliação da APS no Brasil ainda é incerto, uma vez que a avaliação realizada pelo PMAQ-AB, com 893 padrões de qualidade 21, será substituída pelo monitoramento de 21 indicadores de desempenho.

Os resultados deste estudo comprovaram que a APS de alta qualidade teve impacto na redução das internações por condições sensíveis à atenção primária, mesmo em contextos de desigualdade social. Dessa forma, investimentos políticos, institucionais e organizacionais precisam ser feitos a fim de promover a melhora contínua da qualidade dos serviços de APS de todo o país.

\section{Colaboradores}

D. M. Castro, V. B. Oliveira e A. F. Santos contribuiu na concepção do projeto, análise e interpretação dos dados, redação do artigo, revisão crítica relevante do conteúdo intelectual e aprovação final da versão a ser publicada. A. C. S. Andrade contribuiu na análise e interpretação dos dados, redação do artigo, revisão crítica relevante do conteúdo intelectual e aprovação final da versão a ser publicada. M. L. Cherchiglia contribuiu na revisão crítica relevante do conteúdo intelectual e aprovação final da versão a ser publicada.

\section{Informações adicionais}

ORCID: Dayanna Mary de Castro (0000-00028278-0308); Veneza Berenice de Oliveira (00000002-1247-2465); Amanda Cristina de Souza Andrade (0000-0002-3366-4423); Mariângela Leal Cherchiglia (0000-0001-5622-567X); Alaneir de Fátima dos Santos (0000-0002-7674-0449). 


\section{Referências}

1. Rosano A, Loha CA, Falvo R, van der Zee J, Ricciardi W, Guasticchi G, et al. The relationship between avoidable hospitalization and accessibility to primary care: a systematic review. Eur J Public Health 2013; 23:356-60.

2. van Loenen T, van den Berg MJ, Westert GP, Faber MJ. Organizational aspects of primary care related to avoidable hospitalization: a systematic review. Fam Pract 2014; 31:502-16.

3. Ministério da Saúde. Portaria no 221, de 17 de abril de 2008. Diário Ofical da União 2008; 18 abr.

4. Mendonça CS, Leotti VB, Dias-da-Costa JS, Harzheim E. Hospitalizations for primary care sensitive conditions: association with socioeconomic status and quality of family health teams in Belo Horizonte, Brazil. Health Policy Plan 2017; 32:1368-74.

5. Alfradique ME, Bonolo PF, Dourado I, LimaCosta MF, Macinko J, Mendonça CS, et al. Internações por condições sensíveis à atenção primária: a construção da lista brasileira como ferramenta para medir o desempenho do sistema de saúde (Projeto ICSAP - Brasil). Cad Saúde Pública 2009; 25:1337-49.

6. Rasella D, Harhay MO, Pamponet ML, Aquino $\mathrm{R}$, Barreto ML. Impact of primary health care on mortality from heart and cerebrovascular diseases in Brazil: a nationwide analysis of longitudinal data. BMJ 2014; 349:g4014.

7. Aquino R, Oliveira NF, Barreto ML. Impact of the family health program on infant mortality in Brazilian municipalities. Am J Public Health 2009; 99:87-93.

8. Rasella D, Aquino R, Barreto ML. Reducing childhood mortality from diarrhea and lower respiratory tract infections in Brazil. Pediatrics 2010; 126:534-40.

9. Dourado I, Oliveira VB, Aquino R, Bonolo P, Lima-Costa MF, Medina MG, et al. Trends in primary health care-sensitive conditions in Brazil: the role of the Family Health Program (Project ICSAP-Brazil). Med Care 2011; 49:577-84.

10. Macinko J, Oliveira VB, Turci MA, Guanais FC, Bonolo PF, Lima-Costa MF. The influence of primary care and hospital supply on ambulatory care-sensitive hospitalizations among adults in Brazil, 1999-2007. Am J Public Health 2011; 101:1963-70.

11. Mendonça CS, Nedel FB, Batista SR, Medina MG. A utilização do indicador internações por condições sensíveis à atenção primária no brasil. In: Mendonça MHM, Matta GC, Gon$\operatorname{dim}$ R, Giovanella L, organizadores. Atenção primária à saúde no Brasil: conceitos, práticas e pesquisa. Rio de Janeiro: Editora Fiocruz; 2018. p. 527-68.

12. Vuik SI, Fontana G, Mayer E, Darzi A. Do hospitalisations for ambulatory care sensitive conditions reflect low access to primary care? An observational cohort study of primary care usage prior to hospitalization. BMJ Open 2017; 7:e015704.
13. Araujo WRM, Queiroz RCS, Rocha TAH, Silva NC, Thumé E, Tomasi E. Estrutura e processo de trabalho na atenção primária e internações por condições sensíveis. Rev Saúde Pública 2017; 51:75.

14. Mariano TSO. Hospitalização por condições sensíveis à atenção primária em menores de cinco anos de idade em Santa Catarina, 2012. https://repositorio.ufsc.br/xmlui/ handle/123456789/180444 (acessado em 17/ Jan/2019).

15. Cecil E, Bottle A, Ma R, Hargreaves DS, Wolfe I, Mainous AG, et al. Impact of preventive primary care on children's unplanned hospital admissions: a population-based birth cohort study of UK children 2000-2013. BMC Med 2018; 16:151.

16. Pezzin LE, Bogner HR, Kurichi JE, Kwong PL, Streim JE, Xie D, et al. Preventable hospitalizations, barriers to care, and disability. Medicine (Baltimore) 2018; 97:e0691.

17. Tanenbaum J, Cebul RD, Votruba M, Einstadter D. Association of a regional health improvement collaborative with ambulatory care - sensitive hospitalizations. Health Aff (Millwood) 2018; 37:266-74.

18. Gonçalves MR, Hauser L, Prestes IV, Schmidt MI, Duncan BB, Harzheim E. Primary health care quality and hospitalizations for ambulatory care sensitive conditions in the public health system in Porto Alegre, Brazil. Fam Pract 2016; 33:238-42.

19. Afonso MPD, Shimizu HE, Merchan-Hamann E, Ramalho WM, Afonso T. Association between hospitalisation for ambulatory caresensitive conditions and primary health care physician specialisation: a cross-sectional ecological study in Curitiba (Brazil). BMJ Open 2017; 7:e015322.

20. Busby J, Purdy S, Hollingworth W. How do population, general practice and hospital factors influence ambulatory care sensitive admissions: a cross sectional study. BMC Fam Pract 2017; 18:67.

21. Departamento de Atenção Básica, Secretaria de Atenção à Saúde, Ministério da Saúde. Programa Nacional de Melhoria do Acesso e da Qualidade da Atenção Básica (PMAQ): nota metodológica da certificação das equipes. http://189.28.128.100/dab/docs/portaldab/ documentos/nota_metodologica_pmaq.pdf (acessado em 17/Jan/2019).

22. Caminal Homar J, Casanova Matutano C. La evaluacion de La atencion primaria y las hospitalizaciones por ambulatory care sensitive conditions. Marco conceptual. Aten Prim 2003; 31:61-5.

23. Dimitrovová K, Perelman J. Ambulatory care sensitive conditions in Portugal, 2000-2014: socioeconomic inequalities and its costs. Int J Equity Health 2017; 16:145. 
24. Chun, SY, Kim W, Park EC. Disparities in avoidable hospitalization by income in South Korea: data from the National Health Insurance cohort. Eur J Public Health 2019; 29:22531.

25. Busby J, Purdy S, Hollingworth W. A systematic review of the magnitude and cause of geographic variation in unplanned hospital admission rates and length of stay for ambulatory care sensitive conditions. BMC Health Serv Res 2015; 15:324.

26. Macinko J, Dourado I, Aquino R, Bonolo PF, Lima-Costa MF, Medina MG, et al. Major expansion of primary care in Brazil linked to decline in unnecessary hospitalization. Health Aff (Millwood) 2010; 29:2149-60.

27. Pazó RG, Frauches DO, Maria CBM, Cade NV. Modelagem hierárquica de determinantes associados a internações por condições sensíveis à atenção primária no Espírito Santo, Brasil. Cad Saúde Pública 2014; 30:1891-902.

28. Mendonça CS, Harzheim E, Duncan BB, Nunes LN, Leyh W. Trends in hospitalizations for primary care sensitive conditions following the implementation of Family Health Teams in Belo Horizonte, Brazil. Health Policy Plan 2012; 27:348-55.

29. Billings J, Anderson G, Newman L. Recent findings on preventable hospitalizations. Health Aff (Millwood) 1996; 15:239-49.

30. Burgdorf F, Sundmacher L. Potentially avoidable hospitaal admissions in Germany: an analysis of factors influencing rates of ambulatory care sensitive hospitalizations. Dtsch Arztebl Int 2014; 111:215-23.
31. Pinto LF, Giovanella L. Do Programa à Estratégia Saúde da Família: expansão do acesso e redução das internações por condições sensíveis à atenção básica (ICSAB). Ciênc Saúde Coletiva 2018; 23:1903-14.

32. Nedel FB, Facchini LA, Martín M, Navarro A. Características da atenção básica associadas ao risco de internar por condições sensíveis à atenção primária: revisão sistemática da literatura. Epidemiol Serv Saúde 2010; 19:61-75.

33. Silva EN, Powell-Jackson T. Does expanding primary healthcare improve hospital efficiency? Evidence from a panel analysis of avoidable hospitalisations in 5506 municipalities in Brazil, 2000-2014. BMJ Glob Health 2017; 2:e000242.

34. Ministério da Saúde. Portaria no 2.979, de 12 de novembro de 2019. Institui o Programa Previne Brasil, que estabelece novo modelo de financiamento de custeio da Atenção Primária à Saúde no âmbito do Sistema Único de Saúde, por meio da alteração da Portaria de Consolidação no 6/GM/MS, de 28 de setembro de 2017. Diário Oficial da União 2019; 13 nov.

35. Costa FF, Calvo MCM. Avaliação da implantação da Estratégia Saúde da Família em Santa Catarina em 2004 e 2008. Rev Bras Epidemiol 2014; 17:557-70. 


\section{Abstract}

This study aimed to analyze the association between quality of primary healthcare (PHC) in Brazilian municipalities (counties) and the number of hospitalizations due to primary healthcaresensitive conditions. This was an ecological study with analysis of nationwide secondary data. The quality of the number of hospitalizations due to primary healthcare-sensitive conditions was based on assessment of the National Program for Improvement of Access and Quality of Basic Care (PMAQ-AB). The analysis used a hierarchical explanatory model, with the number of the number of hospitalizations due to primary healthcaresensitive conditions hospitalizations in the year 2014 as the dependent variable and sociodemographic and health system data as the independent variables. The measure of association between the number of hospitalizations and quality of PHC was calculated with negative binomial regression with robust variance and the total population as offset, with significance set at $20 \%$ in the univariate analysis and 5\% in the multivariate analysis. The mean number of hospitalizations due to primary healthcare-sensitive conditions admissions during the target period was 359.97 hospitalizations per municipality. The quality of PHC showed a negative association with the number of hospitalizations due to primary healthcare-sensitive conditions admissions. Municipalities with lower quality of PHC (quartile 1) showed 21.2\% more number of hospitalizations due to primary healthcare-sensitive conditions admissions than municipalities with higher quality (95\%CI: 1.091.34). The results showed that quality of PHC in Brazil reduced hospitalizations due to primary healthcare-sensitive conditions, even in contexts of social vulnerability.

Health Policy; Health Services Research; Primary Health Care; Ambulatory Care; Quality of Health Care

\section{Resumen}

El objetivo fue analizar la asociación entre la calidad de la atención primaria a la salud (APS) de los municipios brasileños y el número de internamientos por condiciones delicadas de salud en la atención primaria (ICSAP por sus siglas en portugués). Se trata de un estudio ecológico, con análisis de datos secundarios de alcance nacional. La calidad de la APS se midió en base a la evaluación del Programa Nacional de Mejoría de Acceso y Calidad de la Atención Básica (PMAQ-AB). El análisis se realizó mediante un modelo explicativo jerarquizado, siendo considerado el número de internamientos por condiciones delicadas de salud en la atención primaria, durante el año de 2014, como variable dependiente, $y$ los datos sociodemográficos, así como los relacionados con el sistema de salud, como variables independientes. La medida de asociación entre el número de hospitalizaciones y la calidad de la APS se calculó por regresión binomial negativa con variancia robusta, y la población total como offset, habiendo sido considerada una significancia de un $20 \%$ en el análisis univariado y un $5 \%$ en el multivariado. La cantidad media de ICSAP durante el período analizado fue de 359,97 internamientos por municipio. La calidad de la APS mostró una asociación negativa con el número de internamientos por condiciones delicadas de salud en la atención primaria. Municipios con menor nivel de calidad (cuartil 1) presentaron un 21,2\% más número de internamientos por condiciones delicadas de salud en la atención primaria que los municipios mejor evaluados (IC95\%: 1,091,34). Los resultados mostraron que la calidad de la APS del país tuvo un impacto en la reducción de los números de internamientos por condiciones delicadas de salud en la atención primaria, incluso en contextos de vulnerabilidad social.

Politica de Salud; Investigación sobre Servicios de Salud; Atención Primaria de Salud; Atención Ambulatoria; Calidad de la Atención de Salud
Recebido em 28/Out/2019

Versão final reapresentada em 18/Abr/2020

Aprovado em 27/Abr/2020 